\title{
Original
}

\section{Effect of the leukotriene receptor antagonist montelukast on orthodontic tooth movement}

\author{
Hossam Asaad ${ }^{1)}$, Rabab Al-Sabbagh'), Darem Al-Tabba'2), and Omar Kujan ${ }^{3,4)}$ \\ 1)Orthodontic Department, Faculty of Dentistry, Hama University, Hama, Syria \\ 2)Department of Public Health, Faculty of Veterinary Medicine, Hama University, Hama, Syria \\ ${ }^{3)}$ School of Dentistry, The University of Western Australia, Nedlands, Australia \\ 4)Oral Pathology Department, Faculty of Dentistry, Hama University, Hama, Syria
}

(Received June 20, 2016; Accepted September 27, 2016)

\begin{abstract}
Asthma is a chronic inflammatory disease, and its prevalence is relatively high among children. Optimal management of asthma often requires longterm pharmacotherapy; however, the effects of these medications on orthodontic treatment is uncertain. We evaluated the effects of the leukotriene LTD $_{4}$ receptor antagonist montelukast on orthodontic tooth movement in an animal model. Eight mongrel dogs were given montelukast for periods up to 4 weeks. An orthodontic force of 150-200 $\mathrm{g}$ was applied to move the second and fourth premolars toward the site of the extracted third premolar. The distance between premolars was measured at baseline and at weeks 1 , 2, and 4. Histological examination with hematoxylineosin staining was used to evaluate osteoclast activity. A slight delay in orthodontic movement and decreased osteoclast activity were observed in the montelukasttreated group, as compared with untreated controls. However, the differences were not statistically significant $(P>0.05)$. Our findings suggest that montelukast use will not interfere with orthodontic treatment of asthma patients. However, these findings require confirmation in clinical studies.
\end{abstract}

Keywords: montelukast; osteoclasts; orthodontic tooth movement; asthma; leukotriene receptor antagonists.

Correspondence to Dr. Omar Kujan, School of Dentistry, The University of Western Australia, 17 Monash Avenue, Nedlands WA6009, Australia

E-mail: omar.kujan@uwa.edu.au

Color figures can be viewed in the online issue at J-STAGE.

doi.org/10.2334/josnusd.16-0482

DN/JST.JSTAGE/josnusd/16-0482

\section{Introduction}

Asthma is a chronic episodic disease in which acute exacerbations are interspersed with asymptomatic periods. It affects about 4 to $10 \%$ of the population, and its burden has increased substantially during the last 20 years, particularly in industrialized countries $(1,2)$. Laboratory analysis of bronchoalveolar lavage fluid from asthmatic patients shows inflammatory reactions characterized by increased numbers of mast cells, epithelial cells, eosinophils, lymphocytes, and inflammatory mediators. Once released, such mediators produce immediate and intense inflammatory reactions $(3,4)$. Asthma pharmacotherapy includes drugs that might affect orthodontic tooth movement $(5,6)$.

Inflammation may be an important factor in initiating bone remodeling during orthodontic tooth movement $(5,6)$. Removal of necrotic tissue and bone resorption in orthodontic tooth movement require recruitment of phagocytic cells such as macrophages, foreign body giant cells, and osteoclasts, to remove necrotic tissues from compressed periodontal ligament sites and adjacent alveolar bone (5). Moreover, osteoclast activity is believed to be a good indicator of orthodontic movement (7).

Leukotrienes (LTs) are potent mediators of inflammation, and excessive production of LTs is associated with asthma pathogenesis $(8,9)$. The cysteinyl leukotrienes (CysLTs) are proinflammatory mediators and include $\mathrm{LTC}_{4}, \mathrm{LTD}_{4}$, and $\mathrm{LTE}_{4}$. CysLT1-R activation contributes to all the symptoms observed in airway allergic diseases $(9,10)$. Montelukast, a highly selective antagonist of CysLT receptors, is widely used for treatment of bronchial 
asthma, primarily as an adjunct to corticosteroids $(11,12)$. In this setting, the therapeutic activity of montelukast is achieved through antagonism of CysLT after release of these mediators, mainly from monocytes/macrophages, eosinophils, mast cells, and basophils, as well as by antiinflammatory activity targeting type 2 helper CD4+ T lymphocytes (12). A study of the effects of leukotriene and montelukast on white blood cells in bone marrow found that leukotriene increased the number of such cells and that the increase was abolished by montelukast use (12).

Because a high number of people with asthma use montelukast - particularly children, who might also need orthodontic treatment - the potential effects of montelukast on orthodontic tooth movement are important. However, the effects of montelukast on orthodontic tooth movement are not well understood.

We hypothesized that montelukast delays orthodontic movement. Therefore, using mongrel dogs as a model, we assessed osteoclast activity to determine the effect of montelukast on orthodontic tooth movement.

\section{Materials and Methods}

All experiments were conducted in strict accordance with the recommendations of the National Institutes of Health and the Research, Care, and Use of Laboratory Animals guidelines (13). This in vivo experimental study was reviewed and approved by the Scientific Review Board of Hama University (Ref: AB 014-11). Eight male mongrel dogs (age, 14 months; mean weight, 23.2 $\pm 1.4 \mathrm{~kg}$ ) were obtained from the Veterinary Medicine College of Hama University. The animals were randomly allocated to a control or montelukast-treated group ( $n=$ 4 each; Table 1). The study was conducted at the animal research laboratory of the Veterinary Medicine College of Hama University, where daily care by a veterinarian was provided for the entire experiment. Animals were exposed to a 12-h/12-h light-dark cycle and fed soft dog food and water to prevent any damage to the experimental orthodontic appliance. Their weight was monitored and maintained throughout the experiment. All experimental procedures were performed under intravenous anesthesia with sodium pentobarbital (25-30 mg/kg of body weight).

In total, 32 orthodontic appliances were used in 32 quadrants of the eight dogs, to move the second and fourth premolars toward the site of the extracted third premolar. The orthodontic appliance was made from bands and cast crowns with soldered metal tubes with hooks. Nickel-titanium closed coil springs were used to move the second premolar toward the site of the extracted third premolar, and a force of 150-200 g was exerted by the closed coil spring (5). Four appliances were cemented, one to each quadrant of each dog (Fig. 1).

All third premolars of all dogs were extracted during anesthesia with sodium pentobarbital $(25 \mathrm{mg} / \mathrm{kg})$. All dogs were monitored daily for 12 weeks, as dog bone requires 4-12 weeks to heal (5). The second and fourth premolars were then cut with a diamond bur, to prepare a better shape for retention of cast crowns and bands. Impressions were taken with alginate, and a laboratory technician fabricated the cast crowns and bands and soldered the tubes to the center of the clinical crown. A vertical mark (width $0.5 \mathrm{~mm}$ ) was made on the cast crowns and bands, for use in measuring the distance between teeth. They were then placed on the teeth with nickel-titanium closed coil springs. Each end of the spring was fixed to a hook with a ligature wire. The spring length corresponding to a contractile force of 150-200 $\mathrm{g}$ was determined with a caliper and strain gauge, and the spring activation was set to that length. Four appliances were placed in each dog (one for each quadrant). The baseline, T0, distance between the second and fourth premolars was measured with a digital caliper (Harbor Freight Tools, Calabasas, CA, USA) with an accuracy of $0.01 \mathrm{~mm}$.

All dogs were subject to follow-up and daily examination, to determine if the orthodontic appliances were effective. In the experimental group, four dogs were given oral montelukast (Montegulair, $10 \mathrm{mg}$ tablets, ASIA Pharmaceutical Industries, Aleppo, Syria), 2 mg/ $\mathrm{kg}$, with food once a day (14) for a period up to 4 weeks.

The dogs were euthanized by $\mathrm{CO}_{2}$ inhalation after 1 week (T1), 2 weeks (T2), or 4 weeks (T4) (Table 1). One dog in each group was euthanized at T1 and T2, and two dogs in each group were euthanized at T4. The distance between the second and fourth premolars was measured at each of these time points in all animals. The maxilla and mandible were dissected, and the removed blocks included the teeth adjacent to the teeth assessed for orthodontic movement.

\section{Histological evaluation}

Two sites (the distal root surface of the second premolar and mesial root surface of the fourth premolar) from each quadrant were examined by histological techniques. A total of 64 specimens were collected (32 from each group). To achieve complete decalcification, each block was fixed in $10 \%$ neutral buffered formalin for $24 \mathrm{~h}$ and then decalcified with $14 \%$ ethylenediaminetetraacetic acid for 21 days. A longitudinal buccal-lingual section was made parallel to the long axis of the tooth, to include the orthodontic pressure site of the tooth root. Specimens were then paraffin-embedded and sectioned for hema- 
Table 1 Description of study groups

\begin{tabular}{lll}
\hline Interval & Experimental group & Control group \\
\hline T1 (week 1) & 1 dog given montelukast. & 1 dog given no medication. \\
& Orthodontic tooth movement observed for 1 week; & Orthodontic tooth movement observed for 1 week; \\
& 8 histological samples collected. & 8 histological samples collected. \\
T2 (week 2) & 1 dog given montelukast. & 1 dog given no medication. \\
& Orthodontic tooth movement observed for 2 weeks; & Orthodontic tooth movement observed for 2 weeks; \\
& 8 histological samples collected. & 8 histological samples collected. \\
T4 (week 4) & 2 dogs given montelukast. & 2 dogs given no medication. \\
& Orthodontic tooth movement observed for 4 weeks; & Orthodontic tooth movement observed for 4 weeks; \\
& 16 histological samples collected. & 16 histological samples collected. \\
\hline
\end{tabular}

toxylin and eosin staining. A histopathologist blinded to the group assignment performed the histopathological analysis of the region from the alveolar crest to the tooth apex on the side of compression. The following variables were analyzed in order to assess inflammation and osteoclast activity. Inflammation was graded as 1-3 (1: healthy; 2: mild inflammation with mono- and polynuclear cell infiltration of the periodontal ligament; 3: moderate to severe inflammation, with infiltrate progression toward hard tissues, ie, the tooth root and alveolar bone). Vasodilation was defined as abnormality of blood vessels in the periodontal ligament, if the vessels were high-caliber and congested. Vasodilation was graded as 1 or 2 (1: $\geq 90 \%$ of vessels with unremarkable changes; 2 : $>10 \%$ of vessels with remarkable changes). In addition, bone resorption was graded as 1-3 (1: absence of multinucleated cells and erosions; 2: multinucleated, osteoclast-like cells and erosions observed at edge of alveolar bone; and 3: osteoclast-like cells and erosions present between alveolar bone and tooth root).

Osteoclast activity was assessed on the side of pressure by counting osteoclasts under $100 \times$ magnification. Large multinucleated cells with eosinophilic cytoplasm were considered osteoclasts when present on the bone surface or in bone-resorptive lacunae (15). Osteoclast activity was classified as 0 : no activity, if no osteoclasts were found, 1: low, if one to five osteoclasts were found, 2: moderate, if six to 10 osteoclasts were found, and 3: high, if more than 10 osteoclasts were found.

\section{Statistical analysis}

As described previously, measurement error was evaluated by using the Dahlberg formula, ME $=\sqrt{ } \sum \mathrm{d}^{2} / 2 \mathrm{n}$, where $\mathrm{n}$ is the number of paired measurements and $\mathrm{d}$ is the deviation between two measurements (16). Measurements were recorded twice, by two independent examiners, for each of 16 randomly selected distances of tooth movement at each time point (T0, T1, T2, T4). The error of the method for measurement of tooth movement was $0.019 \mathrm{~mm}$. The Statistical Package for Social

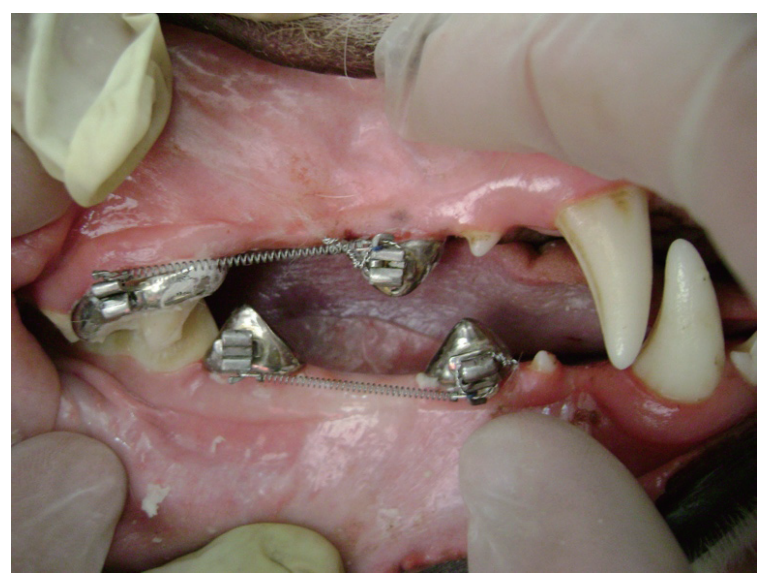

Fig. 1 The orthodontic appliance used in the experiment.

Sciences software version 17.0 (SPSS Inc., Chicago, IL, USA) was used for all statistical analyses, which included the use of descriptive statistics, frequencies/proportions, and the nonparametric Wilcoxon test and paired $t$-test. All statistical tests were two-sided, and $P$ values less than 0.05 were considered to indicate statistical significance.

\section{Results}

In the evaluation of orthodontic movement, the distance between the second and fourth premolars at T2-T4 was shorter in the experimental group than in the control group, but the differences were not statistically significant (Table 2). Table 3 shows the $P$ values for comparisons at all time points (T0, T1, T2, T4). There was no significant difference between the experimental group and control group in mean distances between the second and fourth premolars. These findings suggest that montelukast does not significantly affect the rate of orthodontic tooth movement. Figure 2 shows representative histological sections from the experimental and control groups during the second and fourth weeks of observation. With respect to the effect of montelukast on osteoclast activity, all histological sections from the experimental group showed less osteoclast activity (Fig. 2), but the differences between groups were not significant. Table 4 
Table 2 Difference in mean distance between second and fourth premolars (mm)

\begin{tabular}{lccccc}
\hline & T0-T1 & T0-T2 & T0-T4 & T1-T2 & T2-T4 \\
\hline Experimental group & 0.27 & 0.62 & 0.74 & 0.37 & 0.17 \\
Control group & 0.44 & 0.93 & 1.38 & 0.43 & 0.59 \\
\hline
\end{tabular}

Table 3 Tooth movement in the two study groups (mm)

\begin{tabular}{llllll}
\hline \multirow{2}{*}{ Time } & \multicolumn{2}{l}{ Experimental group } & \multicolumn{2}{c}{ Control group } & \multirow{2}{*}{ v value } \\
& Mean & SD & Mean & SD & \\
\hline T0 & 29.13 & 3.54 & 28.92 & 2.85 & 0.857 \\
T1 & 28.86 & 3.57 & 28.48 & 2.76 & 0.740 \\
T2 & 27.24 & 2.70 & 28.72 & 2.81 & 0.201 \\
T4 & 27.19 & 2.61 & 26.72 & 1.50 & 0.664 \\
\hline
\end{tabular}
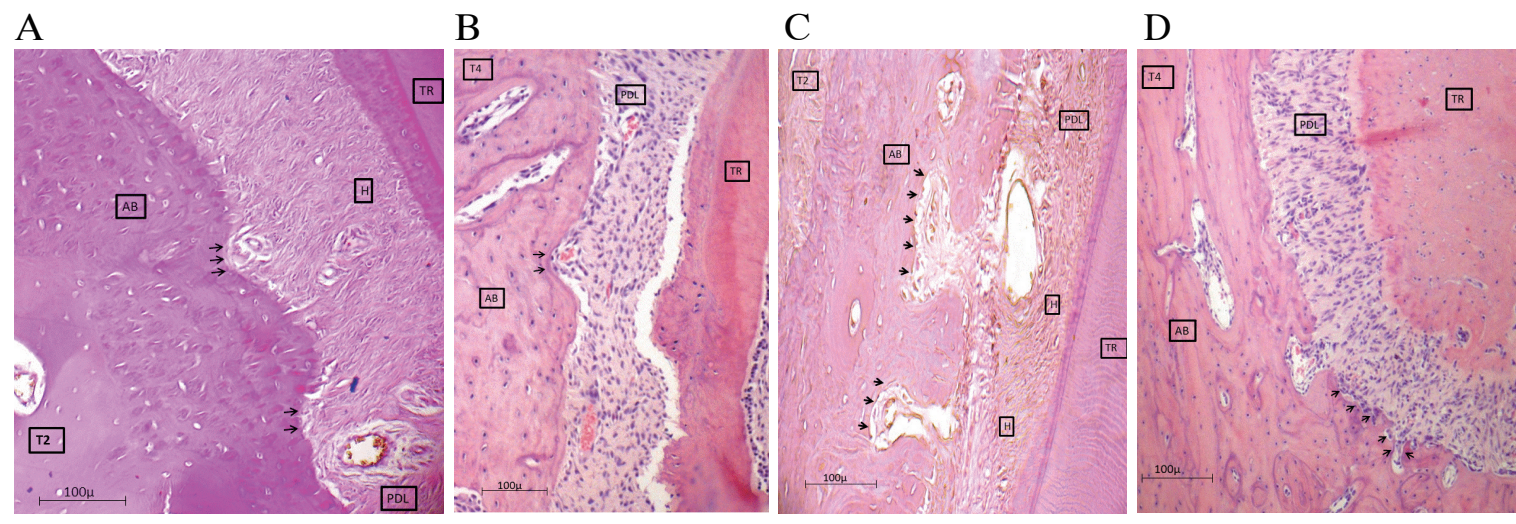

Fig. 2 Photomicrographs of experimental-side periodontal tissue at T2 and T4 in the two study groups. A) Representative section from experimental group at T2 showing osteoclasts in Howship lacunae and hyalinization of PDL; B) representative section from experimental group at T4 showing absence of hyalinization; C) representative section from control group at T2 showing osteoclasts in Howship lacunae; D) representative section from control group at T4 showing complete absence of hyalinization. Arrows show root resorption (hematoxylin and eosin stain, 200×). AB, alveolar bone; PDL, periodontal ligament; TR, tooth root; H, hyalinization; T, number of weeks.

Table 4 Osteoclast activity in the two study groups

\begin{tabular}{llccccc}
\hline & \multicolumn{1}{c}{ Intervals } & No activity & Low activity & Moderate activity & High activity & Total \\
\hline T1 & Experimental group & $0(0 \%)$ & $5(62.5 \%)$ & $3(37.5 \%)$ & $0(0 \%)$ & $8(100 \%)$ \\
& Control group & $0(0 \%)$ & $3(37.5 \%)$ & $2(25.0 \%)$ & $3(37.5 \%)$ & $8(100 \%)$ \\
T2 & Experimental group & $0(0 \%)$ & $7(87.5 \%)$ & $1(12.5 \%)$ & $0(0 \%)$ & $8(100 \%)$ \\
& Control group & $0(0 \%)$ & $5(62.5 \%)$ & $2(25.0 \%)$ & $1(12.5 \%)$ & $8(100 \%)$ \\
T4 & Experimental group & $1(6.3 \%)$ & $11(68.8 \%)$ & $4(25.0 \%)$ & $0(0 \%)$ & $16(100 \%)$ \\
& Control group & $0(0 \%)$ & $8(50.0 \%)$ & $7(43.8 \%)$ & $1(6.3 \%)$ & $16(100 \%)$ \\
\hline
\end{tabular}

shows a comparison of osteoclast activity between study groups at all time points (T1, T2, T4). Table 5 shows the results for the main histological charcteristics, including inflammatory responses during orthodontic movement, such as vasodilation and degree of inflammation. Inflammatory activity was greater in the control group than in the experimental group (Fig. 2); however, the difference was not significant.

\section{Discussion}

We investigated the effects of montelukast under conditions close to those of the human oral environment. Specifically, we used a similar orthodontic force, which was generated by the same open coil springs used for our patients, we allotted sufficient time to complete the orthodontic movement cycle, and we administered the same medication, at the same dose, as that given to asthma patients. 
Table 5 Histological characteristics of the two study groups

\begin{tabular}{|c|c|c|c|c|}
\hline \multirow{2}{*}{ Histological characteristics } & \multirow{2}{*}{ Interval } & \multicolumn{2}{|c|}{ Mean rank } & \multirow{2}{*}{$P$ value } \\
\hline & & Experimental group & Control group & \\
\hline \multirow[t]{3}{*}{ Degree of vasodilation } & $\mathrm{T} 1$ & 7.75 & 9.25 & 0.494 \\
\hline & $\mathrm{T} 2$ & 7.00 & 10.00 & 0.117 \\
\hline & T4 & 14.50 & 18.50 & 0.151 \\
\hline \multirow[t]{3}{*}{ Degree of inflammation } & T1 & 6.88 & 10.13 & 0.120 \\
\hline & $\mathrm{T} 2$ & 9.00 & 8.00 & 0.317 \\
\hline & T4 & 15.00 & 18.00 & 0.151 \\
\hline \multirow[t]{3}{*}{ Osteoclast activity } & $\mathrm{T} 1$ & 6.94 & 10.06 & 0.152 \\
\hline & $\mathrm{T} 2$ & 7.44 & 9.56 & 0.239 \\
\hline & $\mathrm{T} 4$ & 14.13 & 18.88 & 0.098 \\
\hline \multirow[t]{3}{*}{ Degree of bone resorption } & $\mathrm{T} 1$ & 7.50 & 9.50 & 0.317 \\
\hline & $\mathrm{T} 2$ & 8.38 & 8.63 & 0.902 \\
\hline & T4 & 15.41 & 17.59 & 0.415 \\
\hline
\end{tabular}

We observed the characteristic three-phase tooth movement cycle in both groups. Montelukast did not significantly affect orthodontic tooth movement or osteoclast activity and did not yield noteworthy changes in the mean distance between the second and fourth premolars. However, there were nonsignificant differences in the mean distances between premolars, particularly at T4. A slight delay in orthodontic movement, which corresponded with decreased osteoclast activity, was observed in the montelukast intervention group, particularly at T4. This finding might be attributable to the diminishing effect of montelukast on the chemotaxis of lymphocytes and phagocytes and osteoclast differentiation during orthodontic tooth movement. Montelukast has a direct effect on receptor $\mathrm{LTD}_{4}$ and other inflammatory mediators, like prostaglandins, that influence the chemotaxis of phagocytes and osteoclast differentiation $(5,14)$.

Our results are inconsistent with those of some earlier studies of the effect of leukotriene inhibitors and montelukast on orthodontic tooth movement in rats and mice over a period of 12 days $(5,6)$. Mohammed et al found that the leukotriene synthesis inhibitor AA861 significantly inhibited tooth movement in rats (5). However, rats experience continuous incisor eruption, which could affect orthodontic tooth movement. In addition, rat alveolar bone is occasionally aplastic and, as compared with dogs, may require a shorter period for bone resorption and hyalinization during experimental tooth movement (17). In addition, AA861 and montelukast have different potencies. Montelukast is a potent, specific antagonist of leukotriene receptors and decreases production of prostaglandin, which accelerates bone resorption $(18,19)$. Evidence suggests that AA861 causes intracellular redistribution of calcium and increases plasma membrane permeability to calcium uptake. The lipoxygenase pathway, which is triggered by AA861, also increases calcium uptake by platelets (5).

However, our results were consistent with the findings of other studies (6). Using a mouse model, Moura et al. compared the effects of montelukast and the 5-LO inhibitor zileuton on osteoclast activity and orthodontic tooth movement during a 12-day period. Montelukast treatment decreased osteoclast count and orthodontic tooth movement. They concluded that 5-LO-derived metabolites modulate cytokine release and positively affect osteoclast differentiation, thus enhancing bone resorption (6). Baroukh et al studied the effects of the leukotriene inhibitors phenidone and ketoconazole on osteoclasts. After 4 weeks, the numbers and migratory activity of inflammatory cells, particularly phagocytes and osteoclasts, did not differ in hamsters with periodontitis (20).

The limitations of our study include its small sample size and lack of data on leukotriene levels as determined by molecular techniques. A larger sample size and longer period of drug administration and follow-up might yield better evidence. Nevertheless, our study showed that use of a unified sample of mongrel dogs is a good model for studying the effects of medications on orthodontic tooth movement.

In conclusion, montelukast might have a small effect on the rate and extent of orthodontic tooth movement. In addition, osteoclast activity and bone resorption are affected by montelukast use. However, the differences observed were nonsignificant. Future studies should use advanced techniques, such as molecular assays, to investigate the long-term effects of montelukast in humans. In addition, patients should be informed that montelukast use might slightly prolong orthodontic treatment.

\section{Conflict of interest}

None declared. 


\section{References}

1. Loftus PA, Wise SK (2016) Epidemiology of asthma. Curr Opin Otolaryngol Head Neck Surg 24, 245-249.

2. Lochte L, Nielsen KG, Petersen PE, Platts-Mills TA (2016) Childhood asthma and physical activity: a systematic review with meta-analysis and Graphic Appraisal Tool for Epidemiology assessment. BMC Pediatr 16, 50.

3. Ciolkowski J, Mazurek H, Hydzik P, Stasiowska B (2016) Inflammatory markers as exacerbation risk factors after asthma therapy switch from inhaled steroids to montelukast. Pulm Pharmacol Ther 39, 7-13.

4. Durham AL, Caramori G, Chung KF, Adcock IM (2016) Targeted anti-inflammatory therapeutics in asthma and chronic obstructive lung disease. Transl Res 167, 192-203.

5. Mohammed AH, Tatakis DN, Dziak R (1989) Leukotrienes in orthodontic tooth movement. Am J Orthod Dentofacial Orthop 95, 231-237.

6. Moura AP, Taddei SR, Queiroz-Junior CM, Madeira MF, Rodrigues LF, Garlet GP et al. (2014) The relevance of leukotrienes for bone resorption induced by mechanical loading. Bone 69, 133-138.

7. Roberts-Harry D, Sandy J (2004) Orthodontics. Part 11: orthodontic tooth movement. Br Dent J 196, 391-394.

8. Peters-Golden M, Henderson WR Jr (2007) Leukotrienes. N Engl J Med 357, 1841-1854.

9. Crespo A, Giner J, Torrejón M, Belda A, Mateus E, Granel $C$ et al. (2016) Clinical and inflammatory features of asthma with dissociation between fractional exhaled nitric oxide and eosinophils in induced sputum. J Asthma 53, 459-464.

10. Yadav UC, Srivastava SK (2015) Cysteinyl leukotrienes (CysLTs): role in obesity-induced asthma. Curr Mol Med 15, 598-605.

11. Rajanandh MG, Nageswari AD, Ilango K (2015) Assessment of montelukast, doxofylline, and tiotropium with budesonide for the treatment of asthma: which is the best among the second-line treatment? A randomized trial. Clin Ther 37, 418-426.

12. Bush A (2015) Montelukast in paediatric asthma: where we are now and what still needs to be done? Paediatr Respir Rev 16, 97-100.

13. Couto M (2011) Laboratory guidelines for animal care. Methods Mol Biol 770, 579-599.

14. Saito H, Morikawa H, Howie K, Crawford L, Baatjes AJ, Denburg E et al. (2004) Effects of a cysteinyl leukotriene receptor antagonist on eosinophil recruitment in experimental allergic rhinitis. Immunology 113, 246-252.

15. Araújo AS, Fernandes AB, Maciel JV, Netto Jde N, Bolognese AM (2015) New methodology for evaluating osteoclastic activity induced by orthodontic load. J Appl Oral Sci 23, 19-25.

16. Iino $\mathrm{S}$, Sakoda $\mathrm{S}$, Ito $\mathrm{G}$, Nishimori $\mathrm{T}$, Ikeda $\mathrm{T}$, Miyawaki $S$ (2007) Acceleration of orthodontic tooth movement by alveolar corticotomy in the dog. Am J Orthod Dentofacial Orthop 131, 448.

17. Ren Y, Maltha JC, Kuijpers-Jagtman AM (2004) The rat as a model for orthodontic tooth movement--a critical review and a proposed solution. Eur J Orthod 26, 483-490.

18. Leff JA, Busse WW, Pearlman D, Bronsky EA, Kemp J, Hendeles L et al. (1998) Montelukast, a leukotriene-receptor antagonist, for the treatment of mild asthma and exerciseinduced bronchoconstriction. N Engl J Med 339, 147-152.

19. Nimeri G, Kau CH, Abou-Kheir NS, Corona R (2013) Acceleration of tooth movement during orthodontic treatment--a frontier in orthodontics. Prog Orthod 14, 42.

20. Baroukh B, Saffar JL (1992) Leukotriene inhibition in hamster periodontitis. A histochemical and morphometric study. Mediators Inflamm 1, 335-339. 